\section{Oxidative stress in patients with Graves' ophthalmopathy: relationship between oxidative DNA damage and clinical evolution}

\begin{abstract}
Purpose To evaluate the relationship between oxidative stress and clinical evolution in patients with Graves' ophthalmopathy (GO).

Methods Thirty-one euthyroid GO patients and 25 healthy subjects participated in this study. Oxidative DNA damage was assessed by determination of the 8-hydroxy-2'deoxyguanosine (8-OHdG) level in urine by ELISA. The relationship of oxidative DNA damage to the clinical evolutions of GO, especially the smoking status, clinical activity scores (CAS), and ophthalmopathy index was examined.
\end{abstract}

Results The mean 8-OHdG was significantly higher in GO patients than that of normal controls (12.6 \pm 5.7 vs $6.7 \pm 2.5 \mathrm{ng} / \mathrm{mg}$ creatinine, $P<0.001)$. Smokers had significant higher 8-OHdG than did never smokers in GO patients $(P=0.029)$, but not in healthy controls $(P=0.374)$. Among GO patients, only CAS remained significantly correlated with 8-OHdG ( $P=0.001)$ after adjusting for age, sex, disease duration, the status of antithyroid drug and smoking, and thyroid-stimulating hormone level. Patients with active GO (CAS $>3$ ) had higher 8-OHdG than did the patients with CAS $\leqslant 3(16.3 \pm 4.9$ vs $8.6 \pm 3.0$ ng/mg creatinine).

Conclusions Our findings suggest that urinary $8-\mathrm{OHdG}$ is increased in GO patients and is correlated with the disease activity. Smoking had a higher impact on the increased 8-OHdG among GO patients.

Eye (2009) 23, 1725-1730; doi:10.1038/eye.2008.310; published online 10 October 2008
C-C Tsai1,2, C-Y Cheng ${ }^{1,3,4}$, C-Y Liư ${ }^{5}$, S-C Kao', $\mathrm{H}-\mathrm{C} \mathrm{Kau}{ }^{1,6}, \mathrm{~W}-\mathrm{M} \mathrm{Hsu}{ }^{7}$ and $\mathrm{Y}-\mathrm{H} \mathrm{Wei}^{2,5}$

Department of Ophthalmology, Taipei Veterans General Hospital, National Yang-Ming University, Taipei, Taiwan

${ }^{2}$ Institute of Clinical Medicine, National Yang-Ming University, Taipei, Taiwan

${ }^{3}$ Department of Epidemiology, John Hopkins University

Keywords: oxidative stress; Graves' ophthalmopathy; oxidative DNA damage

\section{Introduction}

Graves' ophthalmopathy (GO) affects $25-50 \%$ of patients with Graves' disease that may lead to functional disability and social disturbance, whereas $5 \%$ have potentially sight-threatening disorder. ${ }^{1}$ GO is also known as thyroidassociated ophthalmopathy and thyroid eye disease. Accumulative evidence favours an autoimmune origin with associated histopathological changes of infiltrating mononuclear cells and accumulation of glycosaminoglycans in the orbital connective tissues. ${ }^{2}$ Although many attempts have been made to unravel the pathogenesis of GO from varied viewpoints, much controversy has remained. ${ }^{3-8}$ It may represent a complex interplay of endogenous and environmental factors, and cigarette smoking is probably the most important risk factor in the development and progression of GO. ${ }^{1,9}$ Although a number of potential mechanisms may account for this association, it might also act, in part, by enhancing the generation of reactive oxygen species (ROS) and increasing oxidative stress. ${ }^{10-12}$

More recently, there is growing evidence that oxidative stress plays an important role in the pathogenesis of Graves' disease. ${ }^{13-15}$ Graves' $^{\prime}$ disease is characterized by the overproduction of thyroid hormones, which can accelerate the basic metabolic rate and cellular oxidative metabolism by the induction of mitochondrial enzymes and causes ROS overproduction and

${ }^{5}$ Department of
Bloomberg School of Public Health, Baltimore, MD, USA

${ }^{4}$ Inherited Disease Research Branch, National Human Genome Research Institute, National Institutes of Health, Baltimore, MD, USA Biochemistry and Molecular Biology, National Yang-Ming University, Taipei, Taiwan

${ }^{6}$ Department of Ophthalmology, Koo Foundation Sun Yat-Sen Cancer Centre, Taipei, Taiwan

${ }^{7}$ Department of Ophthalmology, Shuang-Ho Hospital, Taipei Medical University, Taipei, Taiwan

Correspondence: Y-H Wei, Department of Biochemistry and Molecular Biology, School of Medicine, National Yang-Ming University, 155 Li-Nong Street, Section 2, Taipei 112, Taiwan

Tel: + 886228267118 ;

Fax: + 886228264843 .

E-mail: joeman@

ym.edu.tw

Received: 12 May 2008 Accepted in revised form: 15 September 2008 Published online: 10 October 2008

None of the authors have any commercial interest in the material mentioned here 
an increase of oxidative stress. ${ }^{16}$ Bednarek $e t a l^{17}$ reported that the increase in oxidative stress parameters, such as hydrogen peroxide, thiobarbituric acid-reactive substances, superoxide dismutase, and catalase in hyperthyroid patients can be normalized after euthyroidism achievement except those with infiltrative ophthalmopathy. This implies that not only thyroid metabolic status but also ophthalmopathy itself can influence the metabolism of ROS and free radical scavenging enzymes. Several studies provided evidence that ROS are present in the retro-ocular fibroblasts and plasma of patients with GO. ${ }^{18,19}$ However, the contribution of ROS to the pathogenesis of GO has not been clarified yet. In an earlier study, we disclosed that there was a higher urinary 8-hydroxy-2'-deoxyguanosine (8-OHdG) level in eight patients with active GO, and this biomarker of oxidative DNA damage could be reduced after treatment with steroid. ${ }^{20}$ This study was further carried out to investigate the urinary 8-OHdG level in GO patients and evaluated the association between urinary level of 8-OHdG and clinical evolution of GO, to realize more about the role of oxidative stress in the pathophysiology of GO, and hope to open up a potential new avenues for developing novel treatments for GO.

\section{Materials and methods}

\section{Patients}

Thirty-one patients (six men, 25 women, aged $36.1 \pm 12.8$ years, mean \pm SD) with GO were prospectively enroled for this study. Twenty-five age- and sex-matched healthy volunteers (five men, 20 women, aged $38.8 \pm 10.8$ years, mean \pm SD) were recruited as a control group. The diagnosis of GO was based on the criteria proposed by Bartley and Gorman. ${ }^{21}$ Computed tomography of the orbit was carried out to help confirm the diagnosis of GO. Exclusion criteria were any ocular diseases other than GO, alcohol drinking, regular drug ingestion or antioxidant use, pregnancy, as well as individuals suffering from chronic or acute diseases, such as diabetes mellitus, hyperlipidemia, diseases of the lung, liver, or kidney, cancer, other endocrine dysfunction, and immunological or inflammatory disorders. In addition, all study subjects had been not receiving specific treatment (systemic steroids or radiotherapy) or surgical decompression for GO.

To eliminate the influence of abnormal levels of thyroid hormones on the oxidative stress, only the GO patients achieving stable euthyroidism for at least 6 months were included in this study. Thyroid hormone (free T4 and T3), thyroid-stimulating hormone (TSH), and TSH receptor antibody in blood were determined in all GO patients. Ophthalmological investigations included assessment of the activity and severity of GO. The clinical activity of GO was scored according to the clinical activity score (CAS) suggested by Mourits et al, which takes into consideration 10 manifestations of disease; one point is given for any manifestation, and the score may range from 0 (no activity) to 10 (very high activity). ${ }^{22}$ The clinical severity of GO was scored according to the ophthalmopathy index (OI), based on the NOSPECS classification ranging from 0 to 15 points (0-3 points were given for each ocular change based on the severity of soft tissue involvement, proptosis, extraocular muscle involvement, corneal involvement, and sight loss, respectively). ${ }^{23}$ The definition of proptosis mentioned above was adjusted according to the racial variation, and the mean value of exophthalmos in Taiwan normal adults is $13.91 \pm 2.33 \mathrm{~mm} \cdot{ }^{24}$ The study was performed according to the tenets of the Declaration of Helsinki for research involving human subjects. The protocol was approved by the institutional review board of Taipei Veterans General Hospital, and patients gave informed consent for their participation in this study.

\section{Determination of 8-OHdG in urine by ELISA}

Urine samples were freshly obtained from both GO patients and normal healthy subjects. Smokers were requested to abstain from smoking overnight before urine collection. An aliquot of $1 \mathrm{ml}$ urine sample from each subject was centrifuged at $4000 \mathrm{~g}$ for $10 \mathrm{~min}$ at $4^{\circ} \mathrm{C}$ and the supernatant was stored at $-70^{\circ} \mathrm{C}$ until the ELISA analysis. The content of 8-OHdG in urine was measured with an ELISA kit (8-OHdG check, Japan Institute for the Control of Aging, Fukuroi, Japan). Assays were performed according to the manufacturer's instructions. The specificity of the assay had been established in our laboratory and the detection range was $0.5-200 \mathrm{ng} / \mathrm{ml}$. The content of 8-OHdG in the urine of each subject was corrected by creatinine level in urine and is expressed as $\mathrm{ng} / \mathrm{mg}$ creatinine.

\section{Statistical analysis}

Statistical analysis was carried out by using Stata statistical software (StataCorp, College Station, TX, USA). Normality of the data was assessed by the Shapiro-Wilk test. Comparisons between groups were performed with the unpaired Student's $t$-test or the rank-sum test for continuous variables and with the Fisher's exact test or the $\chi^{2}$-test for categorical variables. Correlations between urinary 8-OHdG levels and CAS or OI were determined by Sperman's correlation coefficients. Furthermore, multivariate linear regression was used to assess the association between the urinary 8-OHdG levels and variables of interest, adjusting for potential confounders. 
$P$-values less than 0.05 were considered as statistically significant.

\section{Results}

Table 1 shows the baseline clinical data of patients with GO and normal subjects. No statistical differences in age, sex, and smoking status were observed between the subjects in the two groups. The mean urinary level of 8-OH-dG (ng/mg creatinine) in the GO patients $(12.6 \pm 5.7)$ was significantly higher than that of normal controls $(6.7 \pm 2.5, P<0.001$, Figure 1). The difference remained significant $(P<0.001)$ after adjustment for age, sex, and the smoking status (Table 2). The multivariate regression analysis also showed that GO patients on average had $6.03 \mathrm{ng} / \mathrm{mg}$ creatinine higher urinary 8-OHdG levels than normal controls $(P<0.001)$, and current/ex-smokers on average had $5.23 \mathrm{ng} / \mathrm{mg}$ creatinine higher 8-OHdG levels than did never smokers $(P=0.030)$, irrespective of their case/control status (Table 2). Subgroup analysis showed that current/exsmokers had significant higher $8-\mathrm{OHdG}$ levels than did never smokers in GO patients $(18.5 \pm 6.1$ vs $11.7 \pm 5.1 \mathrm{ng} /$ mg creatinine, $P=0.029)$, but not in healthy controls $(7.6 \pm 2.0$ vs $6.6 \pm 2.6 \mathrm{ng} / \mathrm{mg}$ creatinine, $P=0.374$; Table 3$)$.

Among the $31 \mathrm{GO}$ patients recruited in this study, there were positive correlations between the urinary 8-OHdG level and CAS $(\rho=0.786, P<0.001)$, and between the urinary 8 -OHdG level and OI ( $\rho=0.562$, $P=0.001$ ) (Figures 2 and 3). After adjusting for age, sex, smoking status, disease duration, the status of antithyroid drug, TSH receptor antibody titre, and TSH level, only CAS remained significantly correlated with the urinary level of $8-\mathrm{OHdG}(P=0.001)$, and the correlation between the $8-\mathrm{OHdG}$ level and OI became non-significant $(P=0.389)$. The relationship between the urinary level of $8-\mathrm{OHdG}$ and OI was most severely confounded by the smoking status. In addition, the mean urinary level of $8-\mathrm{OHdG}$ in patients with active GO (CAS $>3$ ) was significantly higher than that of patients with CAS $\leqslant 3(16.3 \pm 4.9$ vs $8.6 \pm 3.0 \mathrm{ng} / \mathrm{mg}$ creatinine, $P<0.001$; Figure 4). We further correlated the $8-\mathrm{OHdG}$ levels to individual CAS parameters and found that the 8-OHdG levels were significantly correlated with (1) painful, oppressive feeling on or behind the globe $(P=0.0051),(2)$ redness of the eyelids $(P=0.0021),(3)$

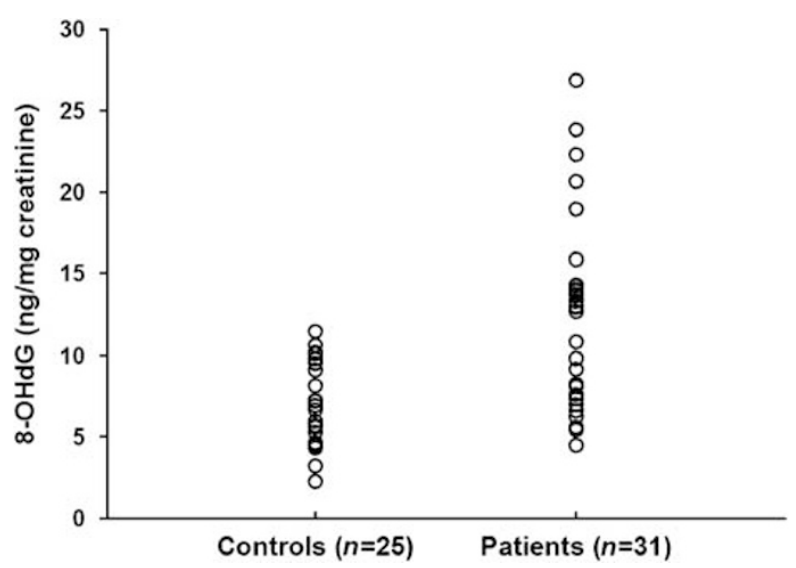

Figure 1 Urinary level of 8-OHdG in normal controls and GO patients.

Table 2 Multivariate linear regression assessing the difference in 8-OHdG levels ( $\mathrm{ng} / \mathrm{mg}$ creatinine) between GO patients and normal subjects

\begin{tabular}{lcrc}
\hline & Coefficient & P-value & $95 \%$ CI \\
\hline Patients vs controls & 6.03 & $<0.001$ & $3.66,8.42$ \\
Age (1 year) & 0.03 & 0.603 & $-0.08,0.13$ \\
Sex (female vs male) & 1.67 & 0.432 & $-2.5,5.91$ \\
Smoking (current/ex-smoker & 5.23 & 0.030 & $0.53,9.93$
\end{tabular}

Table 1 Baseline clinical data of GO patients and normal subjects recruited in this study

\begin{tabular}{lccc}
\hline & Patients $(\mathrm{n}=31)$ & Controls (n=25) & P-value \\
\hline Age (years) & $36.1 \pm 12.8$ & $38.8 \pm 10.8$ & 0.404 \\
Gender (female) & $25(81 \%)$ & $20(80 \%)$ & \\
& & & \\
Smoking status & & $21(84 \%)$ & \\
Never & $27(87 \%)$ & $1(4 \%)$ & 0.952 \\
Ex-smoker & $0(0 \%)$ & $3(12 \%)$ & - \\
Current smoker & $4(13 \%)$ & - & - \\
Mean duration of GO (months) & 7.7 & - & - \\
Under antithyroid drug & $18(58 \%)$ & - & - \\
Mean clinical activity score (range) & $3.4(1-6)$ & & \\
Mean ophthalmopathy index (range) & $4.0(1-7)$ & & \\
\hline
\end{tabular}

${ }^{a}$ Ex-smoker: those had abstained from smoking for more than 6 months. 
Table 3 The mean urinary 8-OHdG levels (ng/mg creatinine) of never smoker and current/ex-smoker among both groups

\begin{tabular}{lrrr}
\hline & Never smoker & current/ex-smoker & P-value \\
\hline GO patients & $11.67 \pm 5.09(n=27)$ & $18.50 \pm 6.05(n=4)$ & 0.029 \\
Normal controls & $6.57 \pm 2.62(n=21)$ & $7.64 \pm 1.97(n=4)$ & 0.374 \\
\hline
\end{tabular}

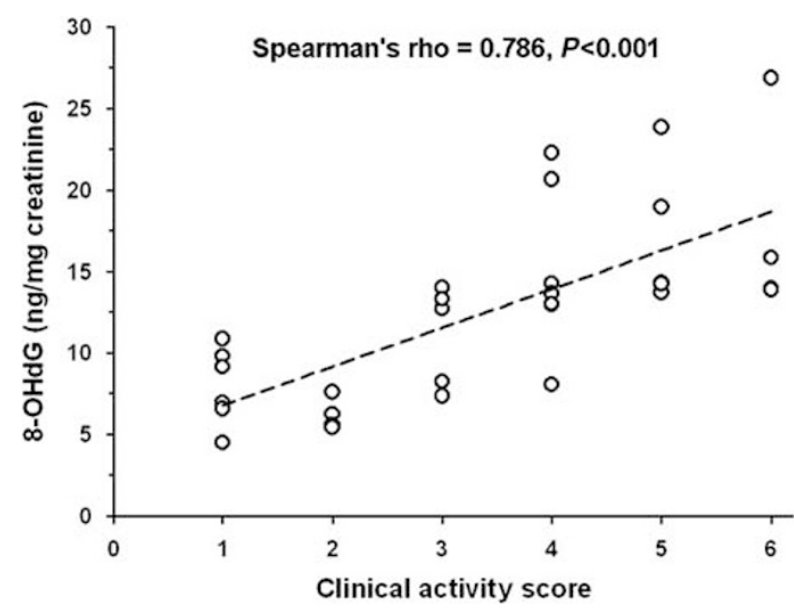

Figure 2 Correlation between urinary level of 8 -OHdG and clinical activity score of $31 \mathrm{GO}$ patients.

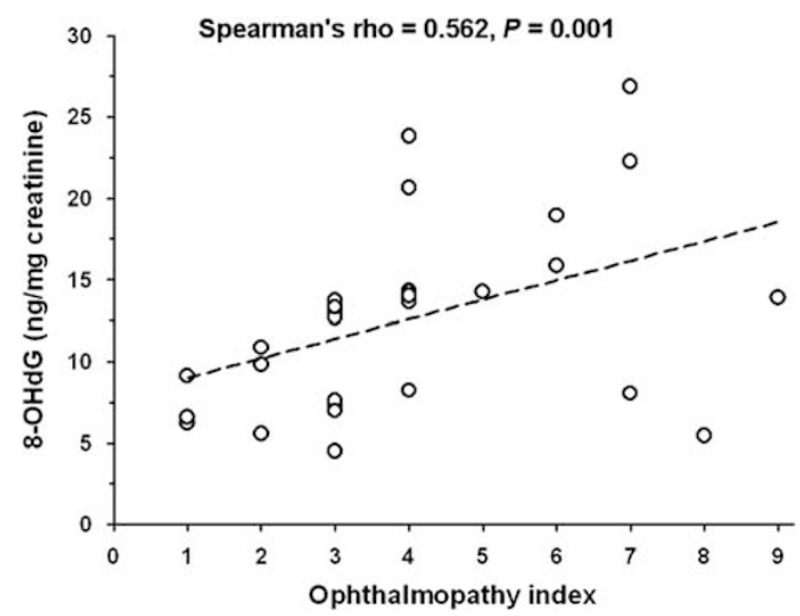

Figure 3 Correlation between urinary level of $8-\mathrm{OHdG}$ and ophthalmopathy index of $31 \mathrm{GO}$ patients.

chemosis (0.0002), and (4) oedema of the eyelids $(P=0.0012)$.

\section{Discussion}

Oxidative stress arises when the production of ROS exceeds the cellular ability to remove ROS and repair cellular damage, and ultimately leads to the widespread oxidative damage to macromolecules, including DNA, lipid and proteins. Oxidative stress appears to be

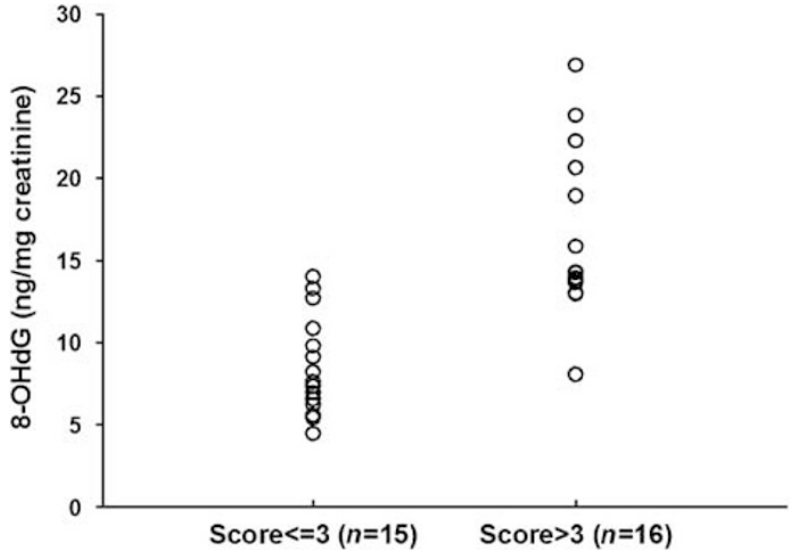

Figure 4 Urinary level of 8-OHdG in GO patients with clinical activity score $\leqslant 3$ and those with clinical activity score $>3$.

involved in a number of pathological conditions, such as degenerative, ${ }^{25}$ neoplastic, ${ }^{26}$ and inflammatory diseases. ${ }^{27}$ Among the assays for oxidative stress, the content of 8-OHdG excreted in urine can be used not only as an indicator of DNA repair capacity, but also as a sensitive biomarker of oxidative DNA damage. An increase in the 8-OHdG level has been observed in patients with various ocular diseases, such as in leukocytes of patients with Eales' disease, ${ }^{28}$ trabecular meshwork of patients with primary open-angle goaucoma ${ }^{29}$ and pterygium tissues. ${ }^{30}$ To our knowledge, this is the first study not only to demonstrate the presence of increased oxidative DNA damage in patients with GO, but also indicate a close relationship between oxidative DNA damage and clinical evolution of GO, particularly the disease activity and smoking.

It has been reported that oxidative stress is present at sites of inflammation, and local inflammatory processes may influence peripheral parameter of oxidative stress. ${ }^{31,32} \mathrm{GO}$ is thought to be an inflammatory disorder of autoimmune background, although the cause is still unknown. In this study, the urinary level of 8-OHdG was found to significantly increase in GO patients (1.9-fold compared with normal subjects), especially in those with active GO (CAS>3) (2.4-fold compared with normal subjects). Moreover, one of our earlier studies demonstrated that patients with active GO on maximal systemic steroid and after treatment had significantly lower urinary level of $8-\mathrm{OHdG}$ (7.19 and $10.18 \mathrm{ng} / \mathrm{mg}$ creatinine, respectively) as compared with those before 
treatment ( $17.47 \mathrm{ng} / \mathrm{mg}$ creatinine), and these changes were accompanied by a decrease of CAS and OI. ${ }^{20}$ In addition, our current findings showed that the urinary 8-OHdG level was closely associated with the CAS of the GO patients. It appears that oxidative DNA damage may be a pathogenic factor, particularly in the inflammatory process of GO. It has also been reported that peripheral markers of ROS were increased in patients with infiltrative GO, and normalization of these markers was observed after intensive corticotherapy. ${ }^{19}$ Thus, it is rational to propose that the ongoing orbital inflammations in patients with GO trigger changes in the levels of ROS, which then causes the subsequent ROSmediated oxidative stress. With respect to the role of oxidative stress in GO, some investigators have provided evidence from basic studies to suggest that oxidative stress is involved in the reactions relevant to GO pathogenesis. Burch et $a l^{33}$ found that superoxide anions could induce a dose-dependent cell proliferation in cultured human retro-ocular fibroblasts from patients with GO, and that such superoxide anion-induced fibroblast proliferation could be prevented by the antioxidant nicotinamide. Hiromatsu et al ${ }^{34}$ also confirmed that nicotinamide could inhibit the activation of orbital fibroblasts induced by interferon- $\gamma$ or tumour necrosis factor- $\alpha$ in fibroblasts from patients with GO. Lu et $a l^{18}$ further revealed that ROS are involved in IL-1- $\beta$ induced glycosaminoglycans accumulation by retroocular fibroblasts. However, further studies are warranted to provide additional information about the precise role of oxidative stress in the pathogenesis of GO.

Cigarette smoking has been demonstrated to induce an increase of oxidative stress status in several chronic diseases. ${ }^{35,36}$ Smoking is also a well-known risk factor for developing GO (odds ratio 7.7), and the odds ratio increase progressively as the severity of eye disease increases. ${ }^{37}$ In this study, we noted that the smokers had higher urinary 8-OHdG levels than did never-smokers among GO patients. In addition, the association between the urinary 8-OHdG levels and the severity of GO became non-significant after adjustment for other parameters, particularly the smoking status. Although the role of smoking in the oxidative stress of GO still needs to be clarified, it may be related to its production of a large quantity of superoxide anions and other ROS, which can induce oxidative stress and perpetuate the ongoing damage in GO. ${ }^{10,33}$ These findings suggest that smoking contributes to not only the severity of eye disease but also an increase of oxidative DNA damage in GO patients, further supporting that cessation of smoking is important in the management of Graves' ophthalmopathy.

The decision of whether ophthalmopathy should be treated often relies on the assessment of the severity and activity of GO, because most GO patients do not require aggressive treatment and tend to improve spontaneously. ${ }^{38,39}$ On the basis of our current and earlier findings, ${ }^{20}$ urinary $8-\mathrm{OHdG}$ can serve as a good biomarker of oxidative stress for GO patients, and such oxidative stress could be relieved by systemic steroid and exacerbated in smokers with GO. In a non-randomized, comparative study, Bouzas et al ${ }^{40}$ showed encouraging results in the treatment of mild and moderately severe GO with oral antioxidants (allopurinol and nicotinamide) for 3 months. However, whether the GO patients, especially those smokers with increased oxidative stress needs specific treatment, such as antioxidants, requires further investigation to clarify the effect of antioxidants on the oxidative stress levels in GO patients.

In conclusion, our results indicate the presence of oxidative DNA damage in urine of patients with GO and a positive correlation between oxidative DNA damage and clinical activity of GO. Smoking had a higher impact on the increased oxidative DNA damage of GO patients than on normal subjects. Oxidative stress may play a role in the pathogenesis of GO, at least in perpetuating the ongoing oxidative damage to DNA as indicated by the level of $8-\mathrm{OHdG}$ in the urine of GO patients.

\section{Acknowledgements}

This study was partially supported by Grants (NSC962314-B-075-023 and NSC96-2320-B-010-006) from the National Science Council of Taiwan and a Grant (V96-B1002) from Taipei Veterans General Hospital, Taipei, Taiwan.

\section{References}

1 Prabhakar BS, Bahn RS, Smith TJ. Current perspective on the pathogenesis of Graves' disease and ophthalmopathy. Endocr Rev 2003; 24: 802-835.

2 Bahn RS, Heufelder AE. Pathogenesis of Graves' ophthalmopathy. N Engl J Med 1993; 329: 1468-1475.

3 Rotella CM, Alvarez F, Kohn LD, Toccafondi R. Graves' autoantibodies to extrathyroidal TSH receptor: their role in ophthalmopathy and pretibial myxedema. Acta Endocrinol 1987; 281: 344-347.

4 Bahn RS. Pathophysiology of Graves' ophthalmopathy: the cycle of disease. J Clin Endocrinol Metab 2003; 88: 1939-1946.

5 Smith TJ, Hoa N. Immunoglobulins from patients with Graves' disease induce hyaluronan synthesis in their orbital fibroblasts through the self-antigen, insulin-like growth factor-I receptor. J Clin Endocrinol Metab 2004; 89: 5076-5080.

6 Smith TJ. The putative role of fibroblasts in the pathogenesis of Graves' disease: evidence for the involvement of the insulin-like growth factor-1 receptor in fibroblast activation. Autoimmunity 2003; 36: 409-415.

7 Chen B, Tsui S, Smith TJ. IL-1 beta induces IL-6 expression in human orbital fibroblasts: identification of an anatomicsite specific phenotypic attribute relevant to thyroidassociated ophthalmopathy. J Immunol 2005; 175: 1310-1319. 
8 Tsai CC, Kau HC, Kao SC, Lin MW, Hsu WM, Liu JH et al. Pulsatile ocular blood flow in patients with Graves' ophthalmopathy. Eye 2005; 19: 159-162.

9 Thornton J, Kelly SP, Harrison RA, Edwards R. Cigarette smoking and thyroid eye disease: a systematic review. Eye 2007; 21: 1135-1145.

10 Pryor WA, Stone K. Oxidants in cigarette smoke. Radicals, hydrogen peroxide, peroxynitrate, and peroxynitrite. Ann N Y Acad Sci 1993; 686: 12-27.

11 Burch HB, Lahiri S, Bahn RS, Barnes S. Superoxide radical production stimulates retroocular fibroblast proliferation in Graves' ophthalmopathy. Exp Eye Res 1997; 65: 311-316.

12 Cawood TJ, Moriarty P, O'Farrelly C, O'Shea D. Smoking and thyroid-associated ophthalmopathy: a novel explanation of the biological link. J Clin Endocrinol Metab 2007; 92: 59-64.

13 Abalovich M, Llesuy S, Gutierrez S, Repetto M. Peripheral parameters of oxidative stress in Graves' disease: the effects of methimazole and 131 iodine treatments. Clin Endocrinol (Oxford) 2003; 59: 321-327.

14 Guerra LN, Ríos de Molina Mdel C, Miler EA, Moiguer S, Karner M, Burdman JA. Antioxidants and methimazole in the treatment of Graves' disease: effect on urinary malondialdehyde levels. Clin Chim Acta 2005; 352: 115-120.

15 Ademoglu E, Ozbey N, Erbil Y. Determination of oxidative stress in thyroid tissue and plasma of patients with Graves' disease. Eur J Intern Med 2006; 17: 545-550.

16 Venditti P, Di Meo S. Thyroid hormone-induced oxidative stress. Cell Mol Life Sci 2006; 63: 414-434.

17 Bednarek J, Wysocki H, Sowinski J. Oxidative stress peripheral parameters in Graves' disease: the effect of methimazole treatment in patients with and without infiltrative ophthalmopathy. Clin Biochem 2005; 38: 13-18.

18 Lu R, Wang P, Wartofsky L, Sutton BD, Zweier JL, Bahn RS et al. Oxygen free radicals in interleukin-1beta-induced glycosaminoglycan production by retro-ocular fibroblasts from normal subjects and Graves' ophthalmopathy patients. Thyroid 1999; 9: 297-303.

19 Bednarek J, Wysocki H, Sowinski J. Peripheral parameters of oxidative stress in patients with infiltrative Graves' ophthalmopathy treated with corticosteroids. Immunol Lett 2004; 93: 227-232.

20 Tsai CC, Kao SC, Cheng CY, Kau HC, Hsu WM, Lee CF et al. Oxidative stress change by systemic corticosteroids treatment of patients with active Graves' ophthalmopathy. Arch Ophthalmol 2007; 125: 1652-1656.

21 Bartley GB, Gorman CA. Diagnostic criteria for Graves' ophthalmopathy. Am J Ophthalmol 1995; 119: 792-795.

22 Mourits MP, Koornneef L, Wiersinga WM, Prummel MF, Berghout A, van der Gaag R. Clinical criteria for the assessment of disease activity in Graves' ophthalmopathy: a novel approach. Br J Ophthalmol 1989; 73: 639-644.

23 Marcocci C, Bartalena L, Bogazzi F, Bruno-Bossio G, Lepri A, Pinchera A. Orbital radiotherapy combined with high dose systemic glucocorticoids for Graves' ophthalmopathy is more effective than radiotherapy alone: results of a prospective randomized study. J Endocrinol Invest 1991; 14: 853-860.

24 Tsai CC, Kau HC, Kao SC, Hsu WM. Exophthalmos of patients with Graves' disease in Chinese of Taiwan. Eye 2006; 20: 569-573.
25 Giacosa A, Filiberti R. Free radicals, oxidative damage and degenerative diseases. Eur J Cancer Prev 1996; 5: 307-312.

26 Federico A, Morgillo F, Tuccillo C, Ciardiello F, Loguercio C. Chronic inflammation and oxidative stress in human carcinogenesis. Int J Cancer 2007; 121: 2381-2386.

27 Tüzün A, Erdil A, Inal V, Aydin A, Bağci S, Yeşilova Z et al. Oxidative stress and antioxidant capacity in patients with inflammatory bowel disease. Clin Biochem 2002; 35: 569-572.

28 Rajesh M, Ramesh A, Ravi PE, Balakrishnamurthy P, Coral $\mathrm{K}$, Punitham R et al. Accumulation of 8-hydroxydeoxyguanosine and its relationship with antioxidant parameters in patients with Eales' disease: implications for antioxidant therapy. Curr Eye Res 2003; 27: 103-110.

29 Sacca SC, Pascotto A, Camicione P, Capris P, Izzotti A. Oxidative DNA damage in the human trabecular meshwork: clinical correlation in patients with primary open-angle glaucoma. Arch Ophthalmol 2005; 123: 458-463.

30 Kau HC, Tsai CC, Lee CF, Kao SC, Hsu WM, Wei YH. Increased oxidative DNA damage, 8-

hydroxydeoxyguanosine, in human pterygium. Eye 2006; 20: 826-831.

31 Koltuksuz U, Uz E, Ozen S, Aydinç M, Karaman A, Akyol O. Plasma superoxide dismutase activity and malondialdehyde level correlate with the extent of acute appendicitis. Pediatr Surg Int 2000; 16: 559-561.

32 Oldenburg B, van Kats-Renaud H, Koningsberger JC, van Berge Henegouwen GP, van Asbeck BS. Chemiluminescence in inflammatory bowel disease patients: a parameter of inflammatory activity. Clin Chim Acta 2001; 310: 151-156.

33 Burch HB, Lahiri S, Bahn RS, Barnes S. Superoxide radical production stimulates retroocular fibroblast proliferation in Graves' ophthalmopathy. Exp Eye Res 1997; 65: 311-316.

34 Hiromatsu Y, Yang D, Miyake I, Koga M, Kameo J, Sato M et al. Nicotinamide decreases cytokine-induced activation of orbital fibroblasts from patients with thyroid-associated ophthalmopathy. J Clin Endocrinol Metab 1998; 83: 121-124.

35 Asami S, Manabe H, Miyake J, Tsurudome Y, Hirano T, Yamaguchi $\mathrm{R}$ et al. Cigarette smoking induces an increase in oxidative DNA damage, 8- hydroxydeoxyguanosine, in a central site of the human lung. Carcinogenesis (London) 1997; 18: 1763-1766.

36 Valko M, Leibfritz D, Moncol J, Cronin MT, Mazur M, Telser J. Free radicals and antioxidants in normal physiological functions and human disease. Int J Biochem Cell Biol 2007; 39: 44-84.

37 Prummel MF, Wiersinga WM. Smoking and risk of Graves' disease. JAMA 1993; 269: 479-482.

38 Perros P, Crombie AL, Kendall-Taylor P. Natural history of thyroid-associated ophthalmopathy. Clin Endocrinol (Oxford) 1995; 42: 45-50.

39 Bartley GB, Fatourechi V, Kadrmas EF, Jacobsen SJ, Ilstrup DM, Garrity JA et al. The treatment of Graves' ophthalmopathy in an incidence cohort. Am J Ophthalmol 1996; 121: 200-206.

40 Bouzas EA, Karadimas P, Mastorakos G, Koutras DA. Antioxidant agents in the treatment of Graves' ophthalmopathy. Am J Ophthalmol 2000; 129: 618-622. 